\title{
The Information-Motivation-Behavioral Skills Model Revisited: A Network-Perspective Structural Equation Model Within a Public Sexually Transmitted Infection Clinic Sample of Hazardous Alcohol Users
}

\author{
Steven A. John ${ }^{1}$ Jennifer L. Walsh ${ }^{2,3,4,5}$ - Lance S. Weinhardt ${ }^{1}$
}

Published online: 3 June 2016

(c) The Author(s) 2016. This article is published with open access at Springerlink.com

\begin{abstract}
The information-motivation-behavioral skills (IMB) model is useful for understanding sexual risk behavior, but has not been tested with hazardously-drinking sexually transmitted infection (STI) clinic patients, a subpopulation at greater HIV risk, or with a network-perspective sexual risk behavior outcome. Participants ( $N=569)$ were STI clinic patients who screened positive for hazardous drinking and risky sexual behavior. Sexual risk behavior (SRB) was operationalized as a latent variable with three indicators: (1) number of sexual partners, (2) number of unprotected sex occasions with primary partner, and (3) number of unprotected sex occasions with non-primary partner(s). Preliminary analyses suggested SRB was best operationalized as a latent variable with two indicators, while unprotected sex with primary partners should be considered separately. In structural models with good fit, the IMB model was generally supported. The IMB model functioned differently for non-primary and multiple
\end{abstract}

Steven A. John

sajohn@uwm.edu

1 Joseph J. Zilber School of Public Health, University of Wisconsin-Milwaukee, P.O. Box 413, Milwaukee, WI 53201-0413, USA

2 Department of Psychiatry and Behavioral Medicine, Center for AIDS Intervention Research (CAIR), Medical College of Wisconsin, Milwaukee, WI, USA

3 Department of Psychiatry and Human Behavior, Alpert Medical School, Brown University, Providence, RI, USA

4 Department of Behavioral and Social Sciences, School of Public Health, Brown University, Providence, RI, USA

5 Center for Behavioral and Preventive Medicine, The Miriam Hospital, Providence, RI, USA partners compared to primary partners in STI clinic patients with hazardous alcohol use.

Keywords IMB model - Behavioral theory · Sexual risk behavior · HIV/AIDS · Hazardous alcohol users

\section{Introduction}

Sexually transmitted infections (STIs) remain highly prevalent within many communities in the United States, and disparities in STIs between Black or African-Americans and Whites keep prevention of STIs a national priority $[1,2]$. STI clinic patients, in particular, are in need of additional attention given their inherent elevated risk for infection and repeat infection [3-5]. Individuals with repeated or untreated infections are at greater risk for medical complications including infertility, ectopic pregnancy, and pelvic inflammatory disease [6]. Patients that continually acquire STIs are also at risk for contracting HIV through continued engagement in sexual risk behavior, and additional evidence suggests an epidemiological synergy with STIs that increases risk for HIV by two- to five-fold regardless of symptomology [7-9].

STI clinic patients classified as hazardous alcohol users represent a subpopulation of STI clinic patients at greater risk for HIV. Physiologically, alcohol use reduces immune function, which makes the likelihood of seroconversion higher upon exposure to HIV [10]. Behaviorally, the causal pathway between alcohol use and sexual risk behavior is less clear. Higher risk of HIV and other STIs in the context of alcohol use could be the result of behavioral disinhibition, decreased condom-use skills, or attitudes during sex after alcohol consumption, but proposed mediating third variables (e.g., personality traits and disorders) further 
muddle causal interpretation [10, 11]. Although research into the causal mechanisms explaining the association between alcohol use and STI risk is still ongoing, alcohol use is associated with increased sexual risk-taking among STI clinic patients [e.g., 12], making interventions for this population of special interest.

Given the limited resources in many STI clinic settings, offering intensive interventions targeting both alcohol risk reduction and sexual risk in addition to providing STI counseling and testing services may not be feasible. For this reason, it is important to identify the key predictors of HIV and STI risk behavior for alcohol-using clinic patients. Testing theory-based models of risk behavior specifically within this sub-population of STI clinic patients may provide direction for researchers and public health practitioners alike. The prevalence of recent alcohol use has been reported as high as $81 \%$ within a large, urban public health STI clinic in the US, with $17 \%$ of those reporting participation within alcohol treatment previously [13]. These findings suggest that alcohol-using STI clinic patients could represent a noteworthy proportion of total patients within some public STI clinics in the US. These patients may be in need of different intervention strategies compared to patients who do not engage in hazardous alcohol use. A more nuanced understanding of how health behavior theories operate for particular high-risk populations can aid in the adaptation or development of population-specific behavioral interventions to be experimentally tested or evaluated within clinical practice settings.

Theory-based research is common in the area of STI and HIV prevention, and many prevention interventions have been based on the information-motivation-behavioral skills (IMB) model [14, 15]. Fisher and Fisher [14] proposed and tested the IMB model based on a critique of previous research, and they argued that risk reduction interventions were most impactful when based on a conceptual framework; population specific; and focused on information, motivation, and behavioral skills. Now frequently used, the IMB model posits that individuals must be informed, motivated, and behaviorally skilled to initiate and maintain HIV prevention behavior. Specifically, individuals must have information that is relevant to the transmission and prevention of STIs and easy to apply in their social setting. Motivation to engage in risk reduction and HIV prevention activities must be supported by individual attitudes and perceived social norms, and highly motivated and informed individuals must have the skills to perform the HIV prevention activity, including self-efficacy, to effectively reduce their risk for HIV and other STIs (see Fig. 1 for conceptual model) [14, 16].

IMB model research is specifically needed with alcoholusing STI clinic patients because theoretical predictors of sexual risk behavior may operate differently with this

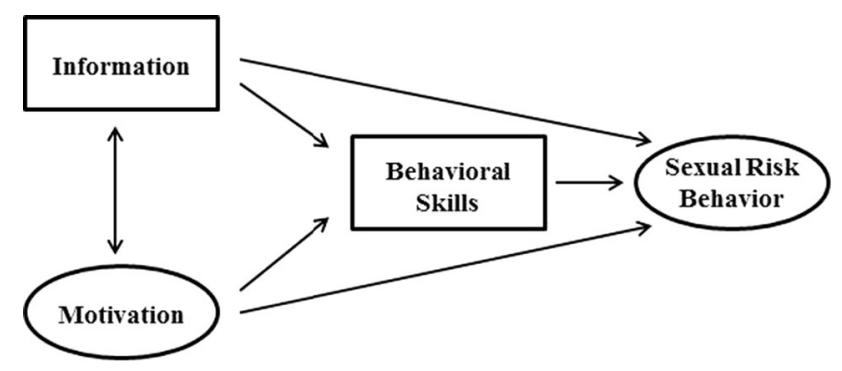

Fig. 1 Information-motivation-behavioral skills conceptual model. Note information-motivation-behavioral skills model adapted from Fisher et al. [56]

unique high-risk population. The IMB model has not been widely used in predicting sexual risk behavior for alcohol users; rather, IMB model antecedents that view intentions as the largest determinant of health behavior [e.g., Theory of Planned Behavior, 17] have been used extensively. For example, researchers find robust evidence in support of an association between alcohol consumption and higher intentions to engage in unprotected sex, which is moderated by heightened sexual arousal, in meta-analytic review [18]. Despite the similarities in theoretical constructs (e.g., attitudes, subjective norms, and intentions) between the IMB model and its antecedents, the IMB model differs in that it does not posit intentions as the largest or most proximal predictor of sexual risk behavior. Intentions are instead a component of the motivational construct of the IMB model that is hypothesized to operate through behavioral skills before behavioral action is taken. Therefore, we believe it is necessary to test the IMB model with this high-risk sample of alcohol users to determine the role of other theoretical constructs-mainly behavioral skillsin mediating the association between intentions and other motivations and sexual risk behavior.

Since conceptualization, the IMB model for HIV prevention has been widely tested using structural equation modeling (SEM), which allows for the use of latent constructs to reduce the influence of measurement error along with the simultaneous consideration of associations between multiple constructs $[19,20]$. However, the measurement of risk is a weakness of prior tests of the IMB model. First, the majority of previous research has included only a single measure of condom use consistency-the percentage of sexual events involving condom use-as an outcome [21-32]. A key limitation of using percentage of condom use is the inability to differentiate levels of risk for individuals who report the same percentage of condom use but have different frequencies of sexual behavior or different types of sexual partners. Second, measuring consistency of condom use without considering number of sexual partners may also lead to an incomplete 
conceptualization of risk that does not account for a more complex understanding of the proximal sexual network.

Previous research has suggested that predictors of unprotected sex may differ for events involving primary versus non-primary partners. For example, Senn et al. [30] found that partner dependence, operationalized as perceived safety, economic, and emotional dependence on a partner, significantly predicted more frequent unprotected sex and a higher proportion of condomless sex with steady (i.e., primary) partners. This study found no significant associations between partner dependence and unprotected sex frequency or proportion with non-steady partners, suggesting the necessity of separately considering sexual risk events with primary and non-primary partners. Additionally, results from prior research have sometimes differed based on whether percentage of condom use or number of unprotected sexual acts is considered as an outcome. For example, Mittal et al. [26] found different results between number and percentage of condom use, particularly between motivation and condom use, across both total sexual events and those specific to steady partners. Although no "gold standard" of measurement has been determined [33], the public health perspective on measurement suggests that each specific unprotected sex event increases risk of STI transmission [34]. A broader conceptualization of risk behavior includes both number of sexual partners and number of unprotected sex events, aligning with a sexual network perspective that places individuals at higher risk for an STI with each additional partner. As such, we use outcome frequency measures to account for proximal sexual network size and each sexual risk event, a conceptual priority within our sexual network perspective.

The current study aims to model the IMB model using a sexual network perspective latent variable. Viewed from a network perspective, sexual risk may differ based on the number of sexual partners, number of unprotected sexual occasions with a primary partner, and number of unprotected sexual occasions with non-primary partner(s). We are not the first to modify the dependent variable in testing the IMB model to account for additional sexual risk measurement. Mustanski et al. [35] modeled a composite indicator of sexual risk for minority youth seeking health services. Within this risk indicator, number of sexual partners and consistency in condom use were measured. Bazargan et al. [36] also modeled a latent dependent variable measuring: (1) ever having sex without condoms, (2) number of sexual partners, and (3) age at first sexual intercourse. Nonetheless, both of these indicators were limited when attempting to account for each specific sexual risk event, a conceptual priority within our sexual network perspective.
Given elevated sexual risk-taking and HIV risk among STI clinic patients who are hazardous alcohol users and the need to identify key intervention targets for this population, we tested the IMB model in a sample attending a Midwestern public STI clinic for confidential HIV counseling and testing. The purpose of this research is not intended to add further evidence to the relationship between alcohol use and sexual risk behavior, but rather to test a theory-based model of risk behavior within a unique population-STI clinic patients with a history of hazardous alcohol use-and perspective using a latent outcome variable with multiple measures of risk. In line with the IMB model, we hypothesized that higher HIV prevention information and motivation would predict higher HIV prevention behavioral skills; higher behavioral skills, in addition to higher information and motivation, would then predict lower sexual risk behavior. Thus, behavioral skills would partially mediate the relationships between information and risk behavior and between motivation and risk behavior. Although we hypothesized direct pathways between all constructs and sexual risk behavior as initially conceptualized by Fisher and Fisher [14], we acknowledged that information may not have a direct association with risk behavior given mixed results from previous IMB models [37]. Key innovations of the current study included the unique, high-risk sample and the multidimensional conceptualization of sexual risk behavior.

\section{Methods}

\section{Participants}

Participants were recruited from a large, Midwestern public STI clinic as part of enrollment into a randomized controlled trial. Research staff determined if individuals were eligible for study recruitment if the participants: (1) were 18 years of age or older; (2) self-reported unprotected vaginal or anal intercourse with two or more sexual partners, an anonymous partner, or an injection drug using partner in the past 3 months, or had been diagnosed and treated for an STI other than HIV in the past 3 months; (3) scored 8 or higher on the AUDIT screening tool for hazardous alcohol use [38, 39]; (4) agreed to a confidential HIV test when offered during standard STI clinical practice; and (5) had no HIV-positive test result in the past.

Of the 1150 patients screened eligible, 606 participants consented to enroll in the study and $N=569$ had complete data used for analysis. Participants had a mean age of 34.41 $(\mathrm{SD}=10.69)$. Seventy percent of participants were male, $89 \%$ Black or African American, and less than $5 \%$ Hispanic. Eighty-six percent of participants had a high school diploma, high school equivalent, or less education, and less 
than $14 \%$ classified as a full- or part-time student. Most participants were unemployed (i.e., $75 \%$ ), and $87 \%$ made less than $\$ 1000$ per month in income. Seventy-three percent of participants were single and never married, and $94 \%$ classified their sexual orientation as heterosexual.

\section{Measurement}

Survey assessments were completed using Audio Computer-Assisted Self-Administered Interviewing (ACASI) software. Scales were used to measure IMB model constructs. Information and behavioral skills were measured as individual indicators with a single scale each, and motivation was measured by scales of condom social norms, condom attitudes, and condom intentions. Dependent variables within our model included count data of sexual partners and unprotected sexual occasions.

\section{Information}

The HIV-KQ-18 [40] was used to measure HIV knowledge, which we operationalized as information. This scale has been validated previously with good internal consistency, test-retest reliability, and valid comparison measures to a longer version HIV knowledge questionnaire, and we found similarly good internal consistency (see Table 1). Participants answered 18 questions with true, false, and don't know responses. Answers were then recoded as correct or incorrect, with all "don't know" responses coded as incorrect. An example question from this scale includes "coughing and sneezing DO NOT spread HIV" [40].

\section{Motivation}

The IMB model construct motivation was measured using three indicator scales of condom social norms, condom attitudes, and condom intentions. Social norms were measured using a 6-item survey with 6-point semantic differential response categories from strongly disagree to strongly agree. An example question includes "current sexual partners think we should use condoms every time." A 10-item scale was used to measure attitudes, and similar response categories of strongly disagree to strongly agree. One example question from this scale is "the use of condoms can make sex more stimulating." Lastly, intentions were measured using a 7-item scale with strongly disagree to strongly agree response categories, and an example question includes "the next time I have sex, I will do only safe sex." Similar indicators for motivation have been used previously within IMB model research $[14,16,41]$.

\section{Behavioral Skills}

HIV prevention self-efficacy, a National Institute of Mental Health Multisite HIV Prevention Trial measure [42], was used to measure behavioral skills within our model. Eight items measured self-efficacy with responses of not at all confident to completely confident scored from 0 to 10 . Gender-specific situational questions were used, and an example question is "how confident are you that you could bring up the issue of condoms or safe sex in a conversation in this situation?" [42]. Self-efficacy has been frequently used as a proxy for behavioral skills within the IMB model literature $[22-25,27,28,30,32,35,36,43-49]$.

\section{Sexual Risk Behavior}

We operationalized our outcome latent variable of sexual risk behavior using three indicators of behavior within the last 90 days: (1) number of sexual partners, (2) number of unprotected sex occasions with primary partner, and (3) number of unprotected sex occasions with non-primary partner(s). The first indicator measured number of sexual

Table 1 Descriptive statistics and reliabilities of model indicators $(N=569)$

\begin{tabular}{|c|c|c|c|c|}
\hline Indicator & Mean & SD & Range & $\alpha^{a}$ \\
\hline HIV-KQ-18 & 13.00 & 3.58 & $0-18$ & 0.80 \\
\hline Social norms & 22.26 & 7.79 & $6-36$ & 0.82 \\
\hline Attitudes & 35.40 & 6.82 & $10-60$ & 0.58 \\
\hline Intentions & 33.33 & 7.31 & $7-42$ & 0.75 \\
\hline Self-efficacy & 59.41 & 18.81 & $0-80$ & 0.91 \\
\hline Number of sexual partners & $4.55(1.26)^{\mathrm{b}}$ & $14.10(0.79)^{\mathrm{b}}$ & $0-300(0-5.71)^{\mathrm{b}}$ & - \\
\hline Number of unprotected sex occasions with primary partner & $25.46(2.21)^{\mathrm{b}}$ & $52.34(1.56)^{\mathrm{b}}$ & $0-1000(0-6.91)^{\mathrm{b}}$ & - \\
\hline Number of unprotected sex occasions with non-primary partner(s) & $5.20(0.98)^{\mathrm{b}}$ & $12.62(1.12)^{\mathrm{b}}$ & $0-99(0-4.61)^{\mathrm{b}}$ & - \\
\hline
\end{tabular}

a Reliability (Cronbach's alpha)

b $\log (\mathrm{x}+1)$ transformed in parentheses

$S D$ standard deviation 
partners separately by gender ["how many different men (women) have you had sex with in the past 90 days?"], and these were combined to form a single indicator. The second and third indicators were created by combining questions measuring vaginal and anal sex occasions separately ["how many times have you had unprotected vaginal (anal) sex with your primary partner in the past 90 days?"; "how many times have you had unprotected vaginal (anal) sex with others in the past 90 days?"].

\section{Statistical Analysis}

Data were prepared using STATA 13.1 (Intercooled), and we analyzed our conceptual model using the operationalized latent variable of sexual risk behavior in Lisrel version 9.1 (Student) using a maximum likelihood estimator [50]. Descriptive statistics and intra-class coefficients using Cronbach's alpha are presented in Table 1 and the variances, covariances, and correlations of indicator variables are presented in Table 2. IMB model indicators were standardized $\left[\left(\mathrm{x}_{\mathrm{i}}-\mathrm{x}_{\text {mean }}\right) / \mathrm{SD}\right]$ to reduce multicollinearity, and outcome variables $\log (\mathrm{x}+1)$ transformed to improve normality. Information and behavioral skills had single indicators as scales, thus we set the error variance to [(1 - intra-class coefficient $) *$ sample variance]. Motivation is estimated using the three indicator variables, as is sexual risk behavior. Motivation and information are allowed to covary. We include direct paths from information and motivation to sexual risk behavior as well as indirect paths from these constructs to behavior through behavioral skills as conceptualized in the IMB model by Fisher and Fisher [14]. Model fit was determined using multiple, established fit indices. Specifically, we used the $\chi^{2}$ badness-of-fit index, root mean square error approximation (RMSEA), non-normed fit index (NNFI), and comparative fit index (CFI) to guide an estimation of overall model fit. We assumed good model fit when the $\chi^{2} /$ $d f$ ratio was 3 or less, RMSEA $\leq 0.05$, NNFI $>0.95$, and $\mathrm{CFI}>0.95$, and nested models were considered significantly different when the $\chi^{2}$ difference test resulted in a $p$ value $\leq 0.05[20,50-52]$.

\section{Results}

\section{Sexual Risk Behavior: Preliminary Model}

We originally conceptualized sexual risk behavior as a latent construct with three indicators: (1) number of sexual partners, (2) number of unprotected sex occasions with primary partner, and (3) number of unprotected sex occasions with non-primary partner(s). However, a preliminary model with adequate but less than ideal fit $\left[\chi^{2}(16)=80.98\right.$, $p<0.01 ; \quad$ RMSEA $=0.085 \quad(0.067-0.103 \quad 90 \% \quad \mathrm{CI})$; $\mathrm{NNFI}=0.852 ; \mathrm{CFI}=0.915]$ had a low factor loading for unprotected sex with primary partners (see Fig. 2), suggesting the construct of sexual risk behavior was not unidimensional. We therefore included unprotected sex with primary partners as a separate outcome variable. Although we anticipated the ability to successfully model sexual risk behavior from a sexual network perspective, our results provided evidence to suggest that a single construct of sexual risk behavior cannot be modeled as a unidimensional measure of risk within this sample. We postulated the low factor loading for primary partners and model misfit to be the result of potential differences in behavioral scripts between primary and non-primary partners. Individuals with multiple partners, and thus non-primary sexual partners, may engage in different behavior and negotiate condom use differently based on an appraisal of risk or relationship closeness $[30,53]$. Therefore, we respecified this model of sexual risk behavior into two separate models of risk: (1) sexual risk behavior with outside partners, and (2) sexual risk behavior with primary partners.

Table 2 Variances, covariances, and correlations of indicator variables

\begin{tabular}{|c|c|c|c|c|c|c|c|c|}
\hline & 1 & 2 & 3 & 4 & 5 & 6 & 7 & 8 \\
\hline 1. HIV-KQ-18 & 0.985 & -0.072 & -0.097 & -0.026 & 0.133 & -0.048 & 0.127 & -0.076 \\
\hline 2. Subjective Norms & -0.071 & 1.000 & 0.247 & 0.453 & 0.335 & -0.083 & -0.277 & -0.231 \\
\hline 3. Attitudes & -0.096 & 0.247 & 1.000 & 0.241 & 0.088 & 0.001 & -0.052 & -0.037 \\
\hline 4. Intentions & -0.025 & 0.453 & 0.241 & 1.000 & 0.294 & -0.105 & -0.196 & -0.170 \\
\hline 5. Self-efficacy & 0.132 & 0.335 & 0.088 & 0.294 & 1.000 & -0.266 & -0.013 & -0.300 \\
\hline 6. Sexual partners & -0.038 & -0.066 & 0.001 & -0.083 & -0.211 & 0.629 & 0.113 & 0.590 \\
\hline 7. Unprotected sex with primary partners & 0.197 & -0.432 & -0.081 & -0.306 & -0.020 & 0.140 & 2.434 & 0.263 \\
\hline 8. Unprotected sex with non-primary partners & -0.085 & -0.258 & -0.041 & -0.191 & -0.336 & 0.524 & 0.460 & 1.255 \\
\hline
\end{tabular}

Covariances in lower left, variances along diagonal, and correlations in upper right italicized; covariances and variances were standardized for variables $1-5$, and variable 1 is not equal to one due to rounding 


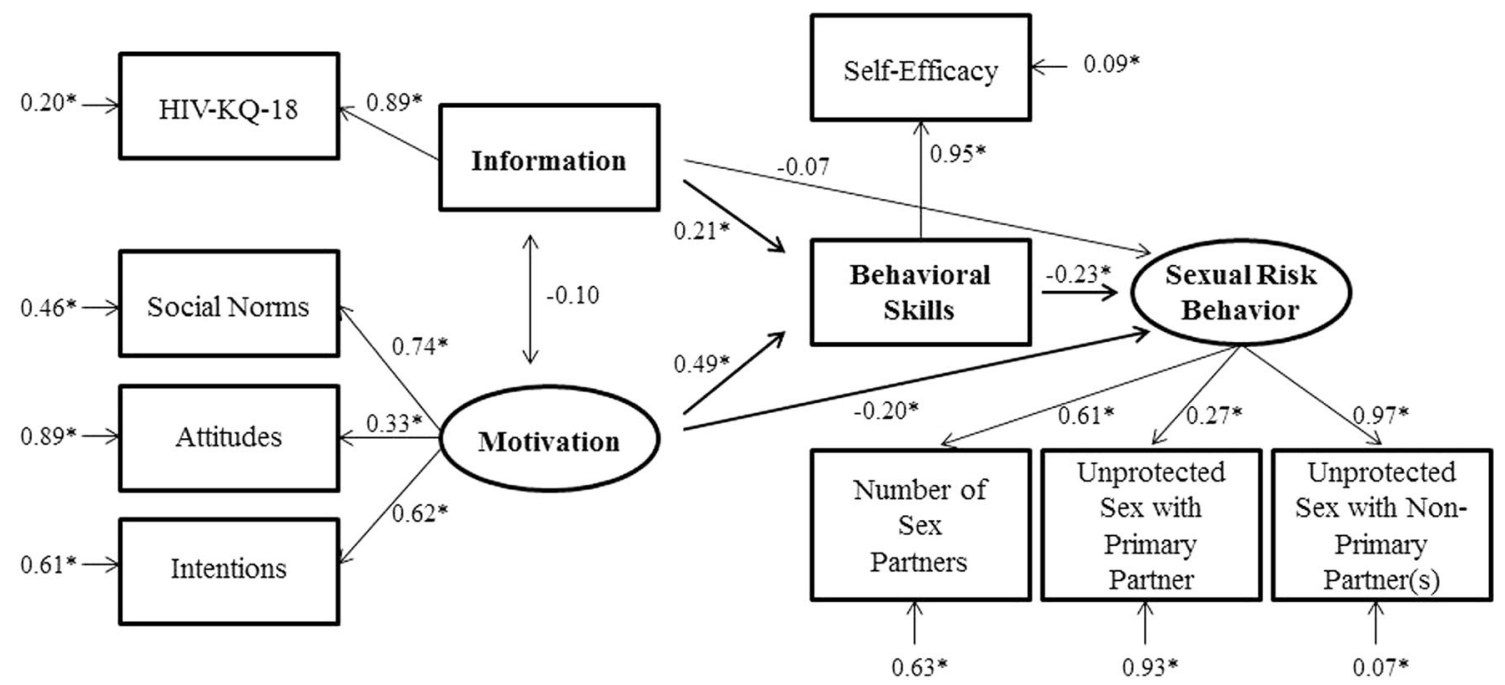

Fig. 2 Preliminary model path diagram completely standardized of the information-motivation-behavioral skills model predicting general sexual risk behavior among STI clinic patients with hazardous alcohol use $(N=569)$. Notes $* p<0.05$; all disturbance terms were

\section{Final Models}

After respecification, the final models retained had good model fit. Specifically, the model of sexual risk behavior with outside partners had acceptable model fit $\left[\chi^{2}(10)=21.42, \quad p=0.02, \quad \chi^{2} / d f \quad\right.$ ratio $=2.14$; RMSEA $=0.045(0.018-0.07190 \% \mathrm{CI}) ; \mathrm{NNFI}=0.963$; $\mathrm{CFI}=0.982]$ and had significantly better fit than our preliminary model $\left[\chi_{\text {diff }}^{2}(6)=59.56, p<0.001\right]$. The path diagram of this first respecified model is illustrated in significant $(p<0.05)$, but removed for interpretation ease; this model had adequate but less than ideal fit $\left[\chi^{2}(16)=80.98, p<0.01\right.$; RMSEA $=0.085 \quad(0.067-0.103 \quad 90 \% \quad$ CI $) ; \quad$ NNFI $=0.852$; $\mathrm{CFI}=0.915]$

Fig. 3. Higher behavioral skills significantly predicted less sexual risk behavior $(\beta=-0.27, p<0.01)$. Behavioral skills fully mediated the association of information with sexual risk behavior $\left(\beta_{\text {indirect }}=0.21, p<0.01\right)$, and partially mediated the association of motivation with sexual risk behavior $\left(\beta_{\text {direct }}=-0.18, p<0.05 ; \beta_{\text {indirect }}=0.49\right.$, $p<0.01)$. Because we were also interested in sexual risk behavior with primary partners, we tested an un-nested comparison model of unprotected sex with primary partners excluding number of sexual partners and unprotected

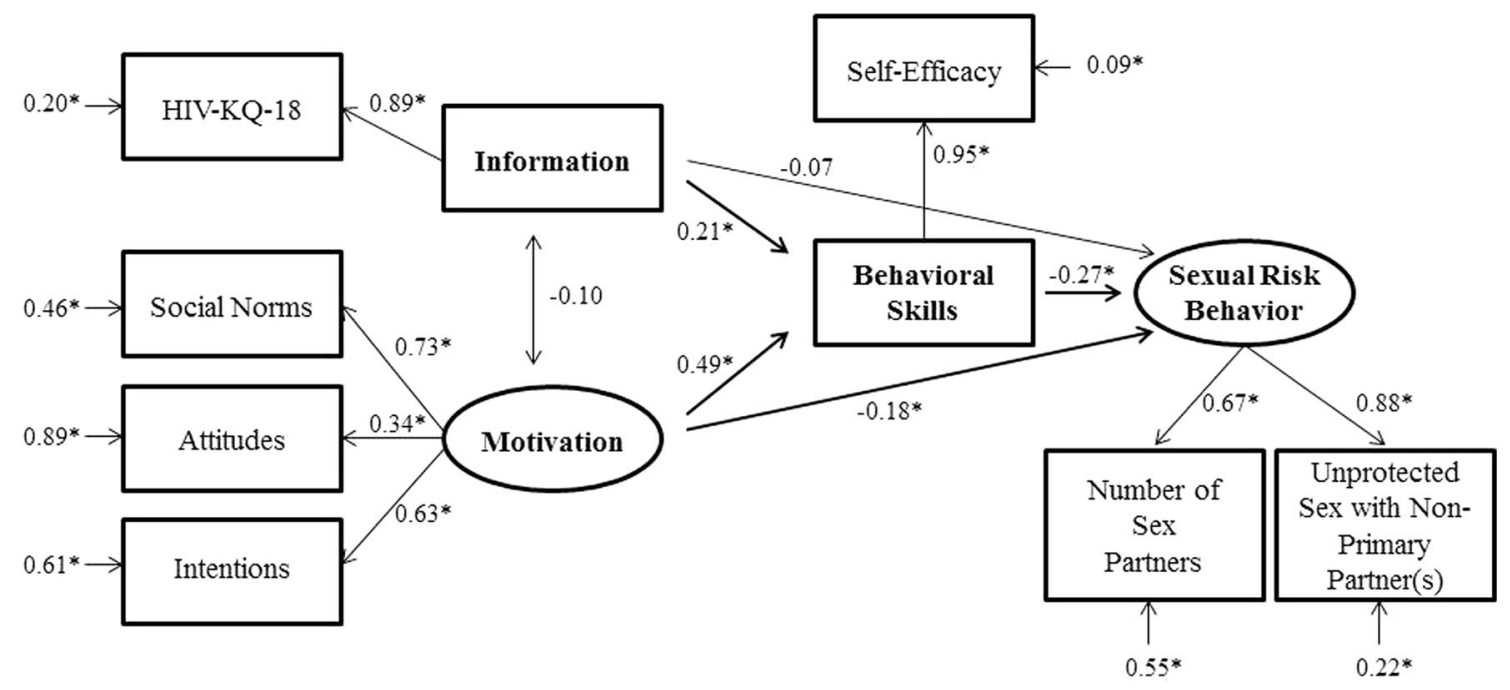

Fig. 3 Path diagram completely standardized of the informationmotivation-behavioral skills model predicting sexual risk behavior with outside partners among STI clinic patients with hazardous alcohol use $(N=569)$. Notes $* p<0.05$; all disturbance terms were significant $(p<0.05)$, but removed for interpretation ease; this model had acceptable fit $\left[\chi^{2}(10)=21.42, p=0.02, \chi^{2} / d f\right.$ ratio $=2.14$; RMSEA $=0.045 \quad(0.018-0.071 \quad 90 \% \quad \mathrm{CI}) ; \quad$ NNFI $=0.963$; $\mathrm{CFI}=0.982]$ 
sex occasions with non-primary partners. The un-nested comparison model had similar acceptable fit $\left[\chi^{2}(6)=12.50, \quad p=0.05, \quad \chi^{2} / d f \quad\right.$ ratio $=2.08$; $\mathrm{RMSEA}=0.044(0.000-0.07890 \% \mathrm{CI})$; NNFI $=0.957$; $\mathrm{CFI}=0.983]$, but the structural model of the IMB constructs changed dramatically. Higher behavioral skills predicted more unprotected sexual occasions with the primary partner $(\beta=0.17, p<0.01)$. Similar to our first final model, behavioral skills fully mediated the association of information with sexual risk behavior $\left(\beta_{\text {indirect }}=0.21\right.$, $p<0.01$ ), and partially mediated the association of motivation with sexual risk behavior $\left(\beta_{\text {direct }}=-0.41\right.$, $\left.p<0.01 ; \beta_{\text {indirect }}=0.49, p<0.01\right)$. This second final model also had significantly better fit than the preliminary model in a nested comparison $\left[\chi_{\text {diff }}^{2}(10)=68.48\right.$, $p<0.001]$. The full path diagram of this second final model is illustrated in Fig. 4, and the standardized beta coefficients, standard errors, and Z-scores for estimates from all models are presented in Table 3.

\section{Discussion}

Our preliminary model of sexual risk behavior using a sexual network perspective resulted in a structural equation model with less than ideal fit. This preliminary analysis modeled a sexual risk behavior latent variable combining three indicators of behavior within the previous 90 days: (1) number of sexual partners, (2) number of unprotected sex occasions with primary partner, and (3) number of unprotected sex occasions with non-primary partner(s). This lack of acceptable model fit was not the result of the IMB model, rather our hypothesized conceptualization of risk. Model fit significantly improved when we separated unprotected sex with primary partners from the other two sexual risk behavior indicators, suggesting low correlation between primary partner risk and the other outcome variables. Another contributing factor to modest model fit within our preliminary model could be the result of a difference in how behavioral skills operated within the IMB model between non-primary and multiple partners compared to primary partners. These findings suggest the IMB model may predict behavior differently for non-primary and multiple partners as compared to primary partners for this high-risk population. Specifically, we found that behavioral skills had the expected negative correlation with risk behavior with non-primary and multiple partners, but a positive association with the number of unprotected sexual acts with main partners. This finding is consistent with some research that suggests different predictors of sexual risk for primary and non-primary partners [30]. Bazargan et al. [22] found that perceiving a monogamous relationship with a partner to be predictive of higher behavioral skills, but a decrease in condom use; behavioral skills may not be protective when looking at unprotected sex with main partners. While we identify some potential congruence of our findings with prior research, additional investigation is needed with alcohol-using STI clinic patients to better understand the association between behavioral skills and unprotected sex with main partners. Specifically,

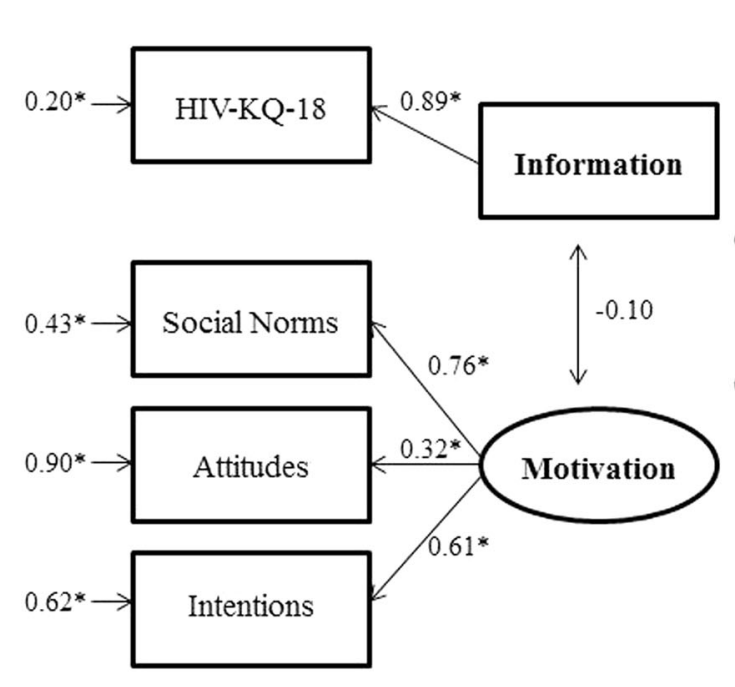

Fig. 4 Path diagram completely standardized of the informationmotivation-behavioral skills model predicting sexual risk behavior with primary partners among STI clinic patients with hazardous alcohol use $(N=569)$. Notes $* p<0.05 ;{ }^{\mathrm{a}}$ single indicator with no

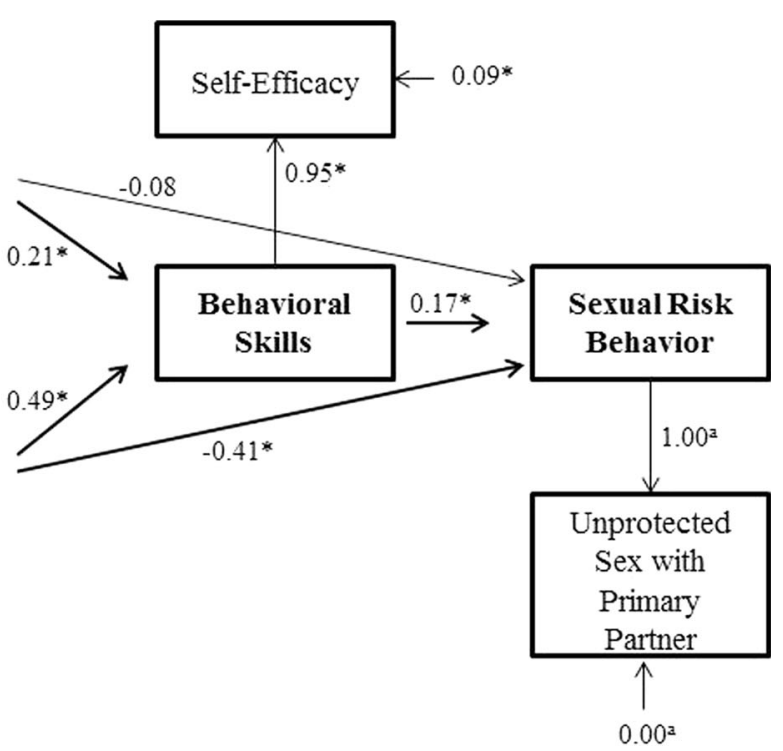

error adjustment; all disturbance terms were significant $(p<0.05)$, but removed for interpretation ease; this model had acceptable fit $\left[\chi^{2}(6)=12.50, \quad p=0.05, \quad \chi^{2} / d f \quad\right.$ ratio $=2.08 ; \quad$ RMSEA $=0.044$ $(0.000-0.07890 \% \mathrm{CI})$; NNFI $=0.957$; CFI $=0.983$ ] 
Table 3 Standardized beta coefficient estimates, standard errors, and z-scores from structural equation models

\begin{tabular}{|c|c|c|c|}
\hline Parameters & Estimate & $\mathrm{SE}$ & $z$-score \\
\hline \multicolumn{4}{|l|}{ Preliminary model } \\
\hline Information $\leftrightarrow$ motivation & -0.102 & 0.038 & -1.765 \\
\hline Information $\rightarrow$ behavioral skills & 0.206 & 0.050 & $4.416 * *$ \\
\hline Motivation $\rightarrow$ behavioral skills & 0.490 & 0.082 & $7.768 * *$ \\
\hline Information $\rightarrow$ sexual risk behavior & -0.070 & 0.027 & -1.428 \\
\hline Motivation $\rightarrow$ sexual risk behavior & -0.199 & 0.045 & $-2.890 * *$ \\
\hline Behavioral skills $\rightarrow$ sexual risk behavior & -0.225 & 0.031 & $-3.667 * *$ \\
\hline Disturbance for information & 1.000 & 0.058 & $13.506^{* *}$ \\
\hline Disturbance for motivation & 1.000 & 0.078 & $7.006 * *$ \\
\hline Disturbance for behavioral skills & 0.738 & 0.053 & $12.673 * *$ \\
\hline Disturbance for sexual risk behavior & 0.860 & 0.032 & $6.248 * *$ \\
\hline \multicolumn{4}{|l|}{ Sexual risk behavior with outside partners } \\
\hline Information $\leftrightarrow$ motivation & -0.102 & 0.038 & -1.759 \\
\hline Information $\rightarrow$ behavioral skills & 0.206 & 0.050 & $4.414 * *$ \\
\hline Motivation $\rightarrow$ behavioral skills & 0.491 & 0.082 & $7.761 * *$ \\
\hline Information $\rightarrow$ sexual risk behavior & -0.069 & 0.031 & -1.314 \\
\hline Motivation $\rightarrow$ sexual risk behavior & -0.177 & 0.052 & $-2.478 *$ \\
\hline Behavioral skills $\rightarrow$ sexual risk behavior & -0.274 & 0.037 & $-4.137 * *$ \\
\hline Disturbance for information & 1.000 & 0.058 & $13.506^{* *}$ \\
\hline Disturbance for motivation & 1.000 & 0.078 & $6.935^{* *}$ \\
\hline Disturbance for behavioral skills & 0.737 & 0.053 & $12.658 * *$ \\
\hline Disturbance for sexual risk behavior & 0.840 & 0.038 & $6.176^{* *}$ \\
\hline \multicolumn{4}{|l|}{ Sexual risk behavior with primary partners } \\
\hline Information $\leftrightarrow$ motivation & -0.102 & 0.038 & -1.770 \\
\hline Information $\rightarrow$ behavioral skills & 0.206 & 0.050 & $4.414 * *$ \\
\hline Motivation $\rightarrow$ behavioral skills & 0.487 & 0.077 & $7.949 * *$ \\
\hline Information $\rightarrow$ sexual risk behavior & 0.075 & 0.084 & 1.555 \\
\hline Motivation $\rightarrow$ sexual risk behavior & -0.412 & 0.145 & $-5.870^{* *}$ \\
\hline Behavioral skills $\rightarrow$ sexual risk behavior & 0.166 & 0.090 & $3.010 * *$ \\
\hline Disturbance for information & 1.000 & 0.058 & $13.506^{* *}$ \\
\hline Disturbance for motivation & 1.000 & 0.076 & $7.536 * *$ \\
\hline Disturbance for behavioral skills & 0.741 & 0.053 & $12.762 * *$ \\
\hline Disturbance for sexual risk behavior & 0.851 & 0.136 & $15.194 * *$ \\
\hline
\end{tabular}

$S E$ standard error

$* p<0.05 ; * * p<0.01$

research considering potential moderators of the association between behavioral skills and sexual risk behavior is called for.

One potential moderator of special relevance to the current population is alcohol consumption within sexual encounters. Behavioral disinhibition from alcohol use could be stronger in sexual encounters with primary partners compared to non-primary partners, moderating the effect between HIV prevention self-efficacy and unprotected sex worthy of additional investigation. The inhibitory cues of higher self-efficacy could be stronger for sexual encounters with non-primary partners regardless of alcohol use, but perceptions of higher self-efficacy could be misinterpreted as confidence in a low-risk unprotected sex event with their primary partner potentially caused by alcohol-related behavioral disinhibition. Kiene et al. [54] applied the alcohol myopia theory [55] to study the moderating factors between condom use self-efficacy and unprotected sex with event-level data to find that alcohol consumption before sex disrupted the inhibitory cues of stronger self-efficacy. Based on our own findings, we suggest future research to determine whether this moderating effect differs based on partnership type.

Our results add to existing literature which found inconsistent results regarding the role of information in the IMB model. Specifically, past studies found that 
information does not always have a direct effect on sexual risk behavior, but many studies suggest that information remains a necessary component of HIV prevention interventions because of the influence knowledge has on behavioral skills. Our results fit with other studies that found that information significantly predicted behavioral skills, but did not directly predict sexual risk behavior $[21,22,24,32,44,48,49,56]$; however, our results conflict with those that found no effect of information [25-28, 31, 35] and those with a direct relationship with condom use [22, 43]. It has been argued that the importance of HIV prevention information may be attenuated within populations with higher levels of knowledge [37], and we found low levels of HIV prevention knowledge within this sample of alcohol-using STI clinic patients providing additional evidence in support of this hypothesis.

The effects of motivation and behavioral skills within our models also provide evidence consistent with many IMB studies, but conflict with others. Our results suggest motivation had a direct effect on behavioral skills, but also had a direct effect on sexual risk behavior. This partial mediation effect of motivation on sexual risk behavior through behavioral skills is consistent with other IMB model research [21, 25, 27-29, 31, 35, 44, 56], but conflicts with evidence of a fully-mediating effect [32, 36, 43, 49]. Thus, motivation and behavioral skills remain important components of the IMB model, but some populations may rely more heavily upon behavioral skills to enact protective behavior compared to others. Our model adds to existing literature suggesting that the IMB model is to be tested within specific populations before planning intervention activities $[14,16]$.

This theory-based research with alcohol-using STI clinic patients may aid researchers and practitioners in adapting and developing further intervention strategies to help this vulnerable population reduce their risk for subsequent STIs including HIV. Although prior research has provided ample support of the IMB model, no previous studies have tested the model with this specific high-risk population. This research allowed us to identify a discrepant finding from other high-risk groups-mainly a difference in how behavioral skills operated based on partnership type. This suggests that interventions targeting self-efficacy for HIV prevention behaviors for patients with primary partners may not be adequate to reduce unprotected sexual behaviors. Instead, additional emphasis should be placed on knowledge, motivation, and potential factors moderating the association between behavioral skills and unprotected sex. In summary, this research prompts additional research into the moderating effects of sexual partnership type between IMB model factors and sexual risk behavior, particularly related to HIV prevention self-efficacy.

The results of this analysis should be interpreted with caution given a number of limitations. First, the cross- sectional nature of this study limits our ability to substantiate any causal effects or rule out any equivalent models, but the findings of our study help support existing evidence published to date. Second, our data are reliant on accurate self-reporting of sensitive behaviors. Although we used ACASI survey methodology to increase the accuracy of our data, we cannot ignore potential response bias. Lastly, the use of self-efficacy as a proxy for behavioral skills could have impacted how behavioral skills operated within the tested IMB model. Although the use of self-efficacy is common within IMB model literature, our finding that HIV-prevention self-efficacy predicted more unprotected sex with primary partners potentially limits our immediate intervention planning abilities to reduce sexual behavior with primary partners using the IMB model. In addition to more focused efforts on knowledge and motivations, intervention strategies based on different theoretical models should also be considered to address risk behavior with primary partners.

Despite these limitations, this research has several noteworthy strengths. First, we were the first to empirically test the IMB model with a multidimensional conceptualization of sexual risk that includes both number of partners and counts of unprotected sexual acts with different partner types. Our strategy allowed us to account for each sexual risk event and sexual partner through frequency measures, a conceptual priority within our sexual network perspective. Although we were unable to identify a unidimensional measure of risk with this specific high-risk population, we recommend additional research with other populations because of the potential public health implications of using a sexual network perspective-mainly a latent observation of risk that incorporates the number of sexual partners and each unprotected sex event. Second, we were the first to empirically test the IMB model within hazardous alcohol users seeking HIV counseling and testing, a specific subpopulation of STI clinic patients with noteworthy risk. Specifically, we found that the IMB model was supported within this sample of alcohol-using STI clinic patients. Moreover, the theoretical components of our IMB models match previous research conducted in this STI clinic that found success in reducing STIs at 1-year follow-up assessments through the use of a full IMB model intervention, as compared to deconstructed intervention components, for risk reduction counseling [13].

\section{Conclusions}

In this study, we found empirical support for the IMB model using a multidimensional conceptualization of sexual risk behavior among hazardous alcohol users seeking HIV counseling and testing within a Midwestern 
public STI clinic. Our findings suggest the IMB model functions differently for non-primary and multiple partners compared to primary partners in STI clinic patients with hazardous alcohol use. Intervention strategies should incorporate these findings into planning interventions for STI clinic patients engaging in hazardous alcohol use to reduce their risk for subsequent STIs including HIV. Alternative theoretical models, including adaptations of the IMB model and exploration of moderating factors, should also be tested to address risk behavior with primary partners for this specific high-risk population. Our research adds evidence in support of the IMB model as a theory-based model that predicts sexual risk behavior, but additional research is needed to more fully understand the implications of the findings related to sexual risk behavior with primary partners.

Acknowledgments Funding support was provided by the National Institute on Alcohol Abuse and Alcoholism (R01-AA013567; PI: Weinhardt) and the National Institute of Mental Health (R01MH089129; PI: Weinhardt; K01-MH099956; PI: Walsh). We would like to thank the anonymous reviewers of this manuscript whose comments and suggestions greatly improved this paper.

\section{Compliance with Ethical Standards}

Conflict of interest The authors declare that they have no conflict of interest.

Ethical Approval All procedures performed in studies involving human participants were in accordance with the ethical standards of the institutional and/or national research committee and with the 1964 Helsinki declaration and its later amendments or comparable ethical standards.

Informed Consent Informed consent was obtained from all individual participants included in the study.

Open Access This article is distributed under the terms of the Creative Commons Attribution 4.0 International License (http://crea tivecommons.org/licenses/by/4.0/), which permits unrestricted use, distribution, and reproduction in any medium, provided you give appropriate credit to the original author(s) and the source, provide a link to the Creative Commons license, and indicate if changes were made.

\section{References}

1. Healthy People 2020: Sexually Transmitted Diseases. http:// www.healthypeople.gov/2020/topics-objectives/topic/sexually-transmitted-diseases. Updated July 22, 2015. Accessed 22 July 2015.

2. Centers for Disease Control and Prevention. Sexually Transmitted Disease Surveillance 2013. http://www.cdc.gov/std/stats13/ surv2013-print.pdf. Published 2014. Accessed 22 July 2015.

3. Evans C, Das C, Kinghorn G. A retrospective study of recurrent chlamydia infection in men and women: is there a role for targeted screening for those at risk? Int J STD AIDS. 2009;20(3):188-92.
4. Newman LM, Warner L, Weinstock HS. Predicting subsequent infection in patients attending sexually transmitted disease clinics. Sex Transm Dis. 2006;33(12):737-42.

5. Peterman TA, Tian LH, Metcalf CA, et al. High incidence of new sexually transmitted infections in the year following a sexually transmitted infection: a case for rescreening. Ann Intern Med. 2006;145(8):564-72.

6. Leichliter JS, Ellen JM, Gunn RA. STD repeaters: Implications for individual and STD transmission in a population. In: Aral SO, Douglas JM, editors. Behavioral interventions for prevention and control of sexually transmitted diseases. New York: Springer Science + Business Media LLC; 2007.

7. Centers for Disease Control and Prevention (CDC). HIV prevention through early detection and treatment of other sexually transmitted diseases-United States. Recommendations of the advisory committee for HIV and STD prevention. MMWR Recomm Rep. 1998;47(RR-12):1-24.

8. Nusbaum MR, Wallace RR, Slatt LM, Kondrad EC. Sexually transmitted infections and increased risk of co-infection with human immunodeficiency virus. J Am Osteopath Assoc. 2004;104(12):527-35.

9. Wasserheit JN. Epidemiological synergy. Interrelationships between human immunodeficiency virus infection and other sexually transmitted diseases. Sex Transm Dis. 1992;19(2): 61-77.

10. Shuper PA, Neuman M, Kanteres F, Baliunas D, Joharchi N, Rehm J. Causal considerations on alcohol and HIV/AIDS-a systematic review. Alcohol Alcohol. 2010;45(2):159-66.

11. Cook RL, Clark DB. Is there an association between alcohol consumption and sexually transmitted diseases? A systematic review. Sex Transm Dis. 2005;32(3):156-64.

12. Kalichman SC, Cain D, Simbayi LC. Multiple recent sexual partnerships and alcohol use among sexually transmitted infection clinic patients, Cape Town, South Africa. Sex Transm Dis. 2011;38(1):18-23.

13. Kalichman SC, Cain D, Weinhardt L, et al. Experimental components analysis of brief theory-based HIV/AIDS risk-reduction counseling for sexually transmitted infection patients. Health Psychol. 2005;24(2):198-208.

14. Fisher JD, Fisher WA. Changing AIDS-risk behavior. Psychol Bull. 1992;111(3):455-74.

15. Kamb ML, Fishbein M, Douglas JM, et al. Efficacy of risk-reduction counseling to prevent human immunodeficiency virus and sexually transmitted diseases: a randomized controlled trial. Project RESPECT study group. JAMA. 1998;280(13):1161-7.

16. Fisher JD, Fisher WA, Bryan AD, Misovich SJ. Informationmotivation-behavioral skills model-based HIV risk behavior change intervention for inner-city high school youth. Health Psychol. 2002;21(2):177-86.

17. Montano DE, Kasprzyk D. Theory of reasoned action, theory of planned behavior, and the integrated behavioral model. In: Glanz K, Rimer BK, Viswanath K, editors. Health behavior and health education: theory, research, and practice. 4th ed. San Francisco: Jossey-Bass; 2008. p. 68-96.

18. Scott-Sheldon LA, Carey KB, Cunningham K, Johnson BT, Carey MP. MASH Research Team. Alcohol use predicts sexual decision-making: a systematic review and meta-analysis of the experimental literature. AIDS Behav. 2016;20(Suppl 1):19-39.

19. Hoyle RH. Introduction and overview. In: Hoyle RH, editor. Handbook of structural equation modeling. New York: The Guilford Press; 2012. p. 3-16.

20. Kline RB. Principles and practice of structural equation modeling. New York: The Guilford Press; 2011.

21. Anderson ES, Wagstaff DA, Heckman TG, et al. Informationmotivation-behavioral skills (IMB) model: testing direct and 
mediated treatment effects on condom use among women in lowincome housing. Ann Behav Med. 2006;31(1):70-9.

22. Bazargan M, Kelly EM, Stein JA, Husaini BA, Bazargan SH. Correlates of HIV risk-taking behaviors among African-American college students: the effect of HIV knowledge, motivation, and behavioral skills. J Natl Med Assoc. 2000;92(8):391-404.

23. Bryan AD, Fisher JD, Fisher WA, Murray DM. Understanding condom use among heroin addicts in methadone maintenance using the information-motivation-behavioral skills model. Subst Use Misuse. 2000;35(4):451-71.

24. Kalichman S, Malow R, Devieux J, Stein JA, Piedman F. HIV risk reduction for substance using seriously mentally ill adults: test of the information-motivation-behavior skills (IMB) model. Community Ment Health J. 2005;41(3):277-90.

25. Kalichman SC, Simbayi LC, Cain D, Jooste S, Skinner D, Cherry C. Generalizing a model of health behaviour change and AIDS stigma for use with sexually transmitted infection clinic patients in Cape Town, South Africa. AIDS Care. 2006;18(3):178-82.

26. Mittal M, Senn TE, Carey MP. Intimate partner violence and condom use among women: does the information-motivationbehavioral skills model explain sexual risk behavior? AIDS Behav. 2012;16(4):1011-9.

27. Pitpitan EV, Kalichman SC, Garcia RL, Cain D, Eaton LA, Simbayi LC. Mediators of behavior change resulting from a sexual risk reduction intervention for STI patients, Cape Town, South Africa. J Behav Med. 2015;38(2):194-203.

28. Robertson AA, Stein JA, Baird-Thomas C. Gender differences in the prediction of condom use among incarcerated juvenile offenders: testing the information-motivation-behavior skills (IMB) model. J Adolesc Health. 2006;38(1):18-25.

29. Scott-Sheldon LA, Carey MP, Vanable PA, Senn TE, CouryDoniger P, Urban MA. Predicting condom use among STD clinic patients using the information-motivation-behavioral skills (IMB) model. J Health Psychol. 2010;15(7):1093-102.

30. Senn TE, Carey MP, Vanable PA, Coury-Doniger P. Partner dependence and sexual risk behavior among STI clinic patients. Am J Health Behav. 2010;34(3):257-66.

31. Walsh JL, Senn TE, Scott-Sheldon LA, Vanable PA, Carey MP. Predicting condom use using the information-motivation-behavioral skills (IMB) model: a multivariate latent growth curve analysis. Ann Behav Med. 2011;42(2):235-44.

32. Zhang $\mathrm{H}$, Liao M, Nie $X$, et al. Predictors of consistent condom use based on the information-motivation-behavioral skills (IMB) model among female sex workers in Jinan, China. BMC Public Health. 2011;11:113.

33. Noar SM, Cole C, Carlyle K. Condom use measurement in 56 studies of sexual risk behavior: review and recommendations. Arch Sex Behav. 2006;35(3):327-45.

34. Schroder KE, Carey MP, Vanable PA. Methodological challenges in research on sexual risk behavior: I. Item content, scaling, and data analytical options. Ann Behav Med. 2003;26(2):76-103.

35. Mustanski B, Donenberg G, Emerson E. I can use a condom, I just don't: the importance of motivation to prevent HIV in adolescent seeking psychiatric care. AIDS Behav. 2006;10(6):753-62.

36. Bazargan M, Stein JA, Bazargan-Hejazi S, Hindman DW. Using the information-motivation behavioral model to predict sexual behavior among underserved minority youth. J Sch Health. 2010;80(6):287-95.

37. Fisher JD, Fisher WA, Shuper PA. The information-motivationbehavioral skills model of HIV preventive behavior. In: DiClemente RJ, Crosby RA, Kegler MC, editors. Emerging theories in health promotion practice and research. San Francisco: Wiley; 2009. p. 22-63.

38. Allen JP, Litten RZ, Fertig JB, Babor T. A review of research on the alcohol use disorders identification test (AUDIT). Alcohol Clin Exp Res. 1997;21(4):613-9.
39. Reinert DF, Allen JP. The alcohol use disorders identification test (AUDIT): a review of recent research. Alcohol Clin Exp Res. 2002;26(2):272-9.

40. Carey MP, Schroder KE. Development and psychometric evaluation of the brief HIV knowledge questionnaire. AIDS Educ Prev. 2002;14(2):172-82.

41. Fisher JD, Fisher WA, Misovich SJ, Kimble DL, Malloy TE. Changing AIDS risk behavior: effects of an intervention emphasizing AIDS risk reduction information, motivation, and behavioral skills in a college student population. Health Psychol. 1996;15(2):114-23.

42. Murphy DA, Stein JA, Schlenger W, Maibach E. Conceptualizing the multidimensional nature of self-efficacy: assessment of situational context and level of behavioral challenge to maintain safer sex. Health Psychol. 2001;20(4):281-90.

43. Cai Y, Wang Y, Zheng Z, Wang J, Yao W, Ma J. Predictors of reducing sexual and reproductive risk behaviors based on the information-motivation-behavioral skills (IMB) model among unmarried rural-to-urban female migrants in Shanghai, China. PLoS ONE. 2013;8(4):e62787.

44. Cai Y, Ye X, Shi R, et al. Predictors of consistent condom use based on the information-motivation-behavior skill (IMB) model among senior high school students in three coastal cities in China. BMC Infect Dis. 2013;13:262.

45. Eggers SM, Aaro LE, Bos AE, Mathews C, de Vries H. Predicting condom use in South Africa: a test of two integrative models. AIDS Behav. 2014;18(1):135-45.

46. Fisher CM. Adapting the information-motivation-behavioral skills model: predicting HIV-related sexual risk among sexual minority youth. Health Educ Behav. 2012;39(3):290-302.

47. Fisher CM. Are information, motivation, and behavioral skills linked with HIV-related sexual risk among young men who have sex with men? J HIV AIDS Soc Serv. 2011;10(1):5-21.

48. Harman JJ, Amico KR. The relationship-oriented informationmotivation-behavioral skills model: a multilevel structural equation model among dyads. AIDS Behav. 2009;13(2):173-84.

49. Liu Z, Wei P, Huang M, et al. Determinants of consistent condom use among college students in China: application of the information-motivation-behavior skills (IMB) model. PLoS ONE. 2014;9(9):e108976.

50. Hoyle RH, Panter AT. Writing about structural equation models. In: Hoyle RH, editor. Structural equation modeling: concepts, issues, and applications. Thousand Oaks: Sage Publications Inc; 1995. p. $158-76$.

51. McDonald RP, Ho MH. Principles and practice in reporting structural equation analyses. Psychol Methods. 2002;7(1):64-82.

52. West SG, Taylor AB, Wu W. Model fit and model selection in structural equation modelling. In: Hoyle RH, editor. Handbook of structural equation modeling. New York: The Guilford Press; 2012. p. 209-31.

53. Noar SM, Crosby R, Benac C, Snow G, Troutman A. Application of the attitude-social influence-efficacy model to condom use among African-American STD clinic patients: implications for tailored health communication. AIDS Behav. 2011;15(5):1045-57.

54. Kiene SM, Simbayi LC, Abrams A, Cloete A. Alcohol expectancies and inhibition conflict as moderators of the alcoholunprotected sex relationship: event-level findings from a daily diary study among individuals living with HIV in Cape Town, South Africa. AIDS Behav. 2016;20(Suppl 1):60-73.

55. Steele CM, Josephs RA. Alcohol myopia. Its prized and dangerous effects. Am Psychol. 1990;45(8):921-33.

56. Fisher JD, Fisher WA, Williams SS, Malloy TE. Empirical tests of an information-motivation-behavioral skills model of AIDSpreventive behavior with gay men and heterosexual university students. Health Psychol. 1994;13(3):238-50. 